Ann. Zootech., I967, 16 (2), 2I3-216.

NOTE PRÉLIMINAIRE

\title{
ESSAIS DE CONGÉLATION DE SPERME DE BOUC
}

\author{
M. GOFFAUX et J.-M. CORTEEL \\ avec la collaboration technique de $\mathrm{J}$.-C. Tourneur et F. Bariteau \\ Station de Recherches sur la Physiologie de la Reproduction, \\ Centre de Recherches vétérinaires et zootechniques, 37 - Nouzilly \\ Institut national de la Recherche agronomique
}

\section{INTRODUCTION}

Le maintien du pouvoir fécondant du sperme de bouc, conservé à $5^{\circ} \mathrm{C}$ pendant plus d'une dizaine d'heures, pose des problèmes difficiles (CORTEEI, I966) qui ne paraissent avoir été résolus que par l'emploi d'un dilueur complexe (NishikawA, I96I et I965). Aussi s'est-on efforcé d'appliquer au sperme de bouc les techniques de congélation employées avec succès en insémination bovine.

Les tentatives dans ce domaine ont été relativement nombreuses : SMrTH et Polge, I950 ; Humke, I959; Litess et Ostrovski, I960 ; Weissflog, I96I ; WAIdE et Niwa, I96I ; FraSer, I962 ; Vtachos et TSAKALOFF, I963; BONFER'T, I962, I964, I965; LYNGSET et al., I965.

Bien que les auteurs soient apparemment d'accord sur le taux final de glycérol (7 p. IOO), le nombre total de spermatozoïdes à mettre en place (Ioo à 300 millions), la voie d'insémination (intracervicale), les pourcentages de mises bas après une insémination varient entre 3 p. IOo (WEISSFLOG, I96I) et 85,5 p. IO0 (WAIDE etNIWA, I96I).

En présence de cette variabilité, nous avons repris l'étude de la congélation du sperme de bouc en essayant de mettre en lumière l'effet éventuel de la vitesse de refroidissement et celui de la nature du dilueur sur le pourcentage de spermatozoïdes mobiles après congélation.

Au terme des essais comparatifs in vitro, nous avons effectué une épreuve de fécondance in vivo en utilisant les techniques ayant donné les meilleurs résultats in vitro.

\section{MATÉRIEL, ET MÉTHODES}

\section{A - Techniques de base}

Sept boucs des races Alpine, Poitevine et Saanen ont fourni quatre éjaculats par semaine (I éjaculat par prélèvement) du I 7 août au 7 octobre 1965 .

Après la récolte, les éjaculats, maintenus à $+35^{\circ} \mathrm{C}$, recevaient graduellement le volume de dilueur non glycérolé nécessaire pour amener la concentration en spermatozoïdes à 600 millions par millilitre. La réfrigération à $+5^{\circ} \mathrm{C}$ était réalisée en I h $3^{\circ} \mathrm{mn}$. 
Le volume de l'éjaculat prédilué était doublé par l'adjonction $\dot{a}+5^{\circ} \mathrm{C}$ d'un volume égal de dilueur contenant $\mathrm{x} 4 \mathrm{p}$. 100 de glycérol. Le dilueur glycérolé était ajouté en 40 minutes par additions successives de 5 fractions de volumes croissants. Aussitôt après l'adjonction de la dernière fraction la semence était mise en ampoules de verre à raison de $\mathrm{I} \mathrm{ml}$ par ampoule.

La congélation était réalisée dans un bain d'alcool refroidi au moyen de fragments de glace carbonique, la cristallisation débutant en moyenne $5 \mathrm{~h} 30 \mathrm{mn}$ après la récolte. Parvenus à la température de $-79^{\circ} \mathrm{C}$, les échantillons étaient plongés dans l'azote liquide où ils ont été conservés.

Après décongélation par immersion des ampoules dans un bain d'eau à $+35^{\circ} \mathrm{C}$, la détermination du pourcentage de spermatozoïdes mobiles a été effectuée indépendamment par deux techniciens au microscope à contraste de phase.

\section{$\mathrm{B}-$ Facteurs de variation}

\section{a) Dilueurs.}

I. Lait écrémé : ıoo $\mathrm{ml} \mathrm{H}_{2} \mathrm{O}+$ ıo g de poudre de lait de vache totalement délipidé maintenu à $92^{\circ} \mathrm{C}$ pendant so minutes puis refroidi. Pénicilline : 50000 UI et Streptomycine : $0,050 \mathrm{~g}$ pour ıoo $\mathrm{ml}$ de dilueur.

2. Citrate et jaune d'wuf: Jaune d'wuf: $20 \mathrm{ml}$; solution de citrate de sodium $\left(2 \mathrm{H}_{2} \mathrm{O}\right)$ à $2,9 \mathrm{p}$. 100 $80 \mathrm{ml}$. Pénicilline : 50000 UI. Streptomycine : 0,050 g.

b) Diagrammes de congélation.

I. Type lent

$\left\{\begin{array}{l}{ }^{\circ} \mathrm{C} / \mathrm{mm} \text { de }+5^{\circ} \mathrm{C} \text { à }-10^{\circ} \mathrm{C} \\ 2^{\circ} \mathrm{C} / m n d e-10^{\circ} \mathrm{C} \text { a }-17^{\circ} \mathrm{C} \\ 4^{\circ} \mathrm{C} / m n \text { de }-17^{\circ} \mathrm{C} \dot{\mathrm{a}}-79^{\circ} \mathrm{C}\end{array}\right.$

2. Type très lent $\left\{\begin{array}{l}0,17^{\circ} \mathrm{C} / \mathrm{mn} \text { de }+5^{\circ} \mathrm{C} \text { à }{ }^{\circ} \mathrm{C} \\ 0,5^{\circ} \mathrm{C} / \mathrm{mn} \text { de } 0^{\circ} \mathrm{C} \text { à }-5^{\circ} \mathrm{C}\end{array}\right.$

puis mêmes abaissements thermiques que pour le refroidissement du type lent.

c) Épreuves de fécondance.

La semence congelée dont la fécondance a été ćprouvée par insémination artificielle a reçu les traitements $a_{2}$ et $b_{2}$. Les chèvres ont été inséminées une seule fois au cours de l'astrus par dépôt au voisinage immédiat du cervix de I ml de semence décongelée contenant en moyenne I 35 millions de spermatozoïdes vivants montrant une bonne motilité (moyenne égale à 4 , avec une échelle de cotation comprise entre $\circ$ et 5 .

\section{RÉSULTATS}

\section{Io Essais de laboratoire}

TABLEAU I

Moyennes des pourcentages de spermatozoïdes mobiles après congélation

\begin{tabular}{|c|c|c|c|c|}
\hline $\begin{array}{c}\text { Essai } \\
n^{0}\end{array}$ & $\begin{array}{c}\text { Nombre } \\
\text { éjaculats partagés }\end{array}$ & $\begin{array}{l}\text { Diagrammes } \\
\text { de congélation }\end{array}$ & Dilueurs & P. $100 \mathrm{spz}$ mobiles \\
\hline \multirow[t]{2}{*}{1} & \multirow{2}{*}{27} & Lent & Lait écrémé & 18, , $^{\prime}$ \\
\hline & & Trés lent & Iait écrémé & $20,7 *$ \\
\hline \multirow[b]{2}{*}{2} & \multirow{2}{*}{18} & 'Très lent & Lait écrémé & 23,5 \\
\hline & & Très lent & Citrate et jaune d'œuf & $40,7 * *$ \\
\hline
\end{tabular}




\section{$2^{\circ}$ Inséminations}

Les résultats ont été contrôlés I65 jours en moyenne après l'insémination non suivie de retours en ostrus.

A la suite de 168 inséminations effectuées, 25 mise bas seulement ont été enregistrées ( 5 p. I00). Les taux de mise bas partiels par bouc et par inséminateur ne montrent pas de différence appréciable susceptible d'expliquer cet échec par une carence individuelle.

\section{DISCUSSION}

L'examen du tableau I montre qu'après dilution du sperme dans le milieu au lait écrémé la congélation très lente permet une meilleure survie des spermatozoïdes. Par ailleurs, avec le diagramme de congélation du type " très lent », le pourcentage de spermatozoïdes mobiles après décongélation est significativement plus élevé $(\mathrm{P}<$ o,OI) lorsqu'on utilise le citrate jaune d'œuf comme milieu de conservation.

Les mauvais résultats obtenus in vivo paraissent imputables à un défaut de la technique utilisée. Ce défaut réside peut-être dans l'application d'un temps d'équilibration trop long à de la semence diluée dans un milieu contenant une proportion excessive de jaune d'œuf.

On sait en effet que le plasma séminal de bouc contient un enzyme du type phosphatidase A capable d'hydrolyser la molécule de lécithine du jaune d'œuf en un acide gras et une lysolécithine nuisible pour les spermatozoïdes (IRITANI et NIsHIKAWA, I96I, I963; AAMDAL et al., I965). On peut donc penser que la réduction du temps d'équilibration permettrait d'atteinclre la cristallisation avant que la réaction enzymatique soit suffisamment engagée pour porter atteinte aux spermatozoïdes.

Cette hypothèse paraît ne pas tenir compte des très bons résultats obtenus par WaIde et NIWA (I96I) qui ont utilisé un dilueur identique et un temps d'équilibration de I7 heures. IrI'Tani et NishikaWa (I964) ont toutefois montré que la teneur en phosphatidase du plasma séminal de bouc subit des variations individuelles et saisonnières de forte amplitude. Par ailleurs, dans l'essai de WAIDE et NIWA, une mise en place plus profonde de la semence a pu compenser l'altération des spermatozoïdes résultant des effets propres de la congélation.

\section{CONCLUSION}

En tout état de cause, ces essais fournissent une illustration des difficultés qu'on éprouve à préjuger le pouvoir fécondant de la semence de bouc congelé en s'appuyant sur les taux de réanimation obtenus in vitro après décongélation. 


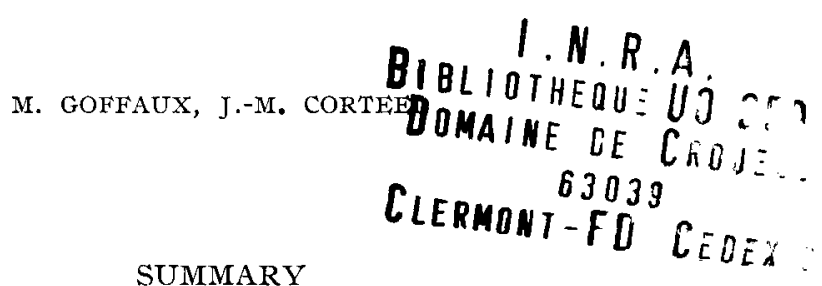

A TRIAL OF DIFFERENT FREEZING PROCESSES FOR GOAT SEMEN.

PRELIMINARY REPORT

The effect of freezing speed and dilution medium on the percent sperm motile after thawing was studied on 45 ejaculates collected from 7,2 to 6 years old goats.

A very slow freezing process $\left(0.17^{\circ} \mathrm{C}\right.$ per minute from $+5^{\circ} \mathrm{C}$ to $0^{\circ} \mathrm{C}: 0.5^{\circ} \mathrm{C}$ per minute from $0^{\circ} \mathrm{C}$ to $-5^{\circ} \mathrm{C}$ ) enabled a better survival of spermatozoa than a $I^{\circ} \mathrm{C}$ per minute freezing down to $-5^{\circ} \mathrm{C}$.

With the first freezing process, the use of a citrate-egg yolk dilutor enabled to obtain a percent motile spermatozoa significantly higher than that obtained with skim-milk.

The insemination of 168 goats with sperm frozen by the best-seeming process induced 25 controlled kiddings. The possible causes of this failure are discussed.

\section{RÉFÉRENCES BIBLIOGRAPHIQUES}

Aamdal J., Lyngset O., K. Fossun. Toxic effect of lysolecithin on sperm. A preliminary report Nord. Vet. Med., 17, 633-634.

BONFERT A., 1962. Erfolgreicher Besamungversuch mit Ziegenfrostsperma. Deulsch. Tier. Wosch., 60, 450-45I.

BONFERT A., I 964 . Ziegenbesamung mit Frostsperma im Saarland. Ve Congrès Internat. Reproduction anim. Insém. artif., Trento., vol. 4, $257^{-26} 3$.

Bonfert A., i965. Communication personnelle.

CoRteer, J. M., 1966. Reproduction et insémination artificielle dans l'espèce caprine. Bull. techn.inform., 210, 463-471.

Fraser A., 1962. A technique for freezing goat semen and results of a small breeding trial. Canad. Vel. J., 3, I33-144.

HUMKE R., 1959. Samenübertragung bei Ziegen, 10. Beitrag. Samenkonservierungs und Besamungsversuche unter besonderer Berücksichtigung des Samentiefkühlverfahrens ( $\left.79^{\circ} \mathrm{C}\right)$. Dissert., Richard Gölze Institut, Hannover.

Iritani A., Nisilikawa Y., I96r. Studies of the egg-yolk coagulating factor in goat semen. I-II. Proc. Silver Jubilee. I.ab. anim. husbandry, Coll. Agric., Kyoto Univ., March, I96r.

IrITANI A., Nishikawa Y., I963. Studies of the egg-yolk coagulating factor in goat semen, III, IV. Jap. J. anim. Reprod., 8, 109-I 17.

IRITANI A., Nishikawa Y., I g64. Studies on the egg-Yolk coagulating enzyme in goat semen. VII. Variations in the enzyme activity of the semen between breeding and non breeding season and in each ejaculate collected three times successively. Jap. J. anim., 10, 52-56.

Liess J., Ostrowski J. E. B., i 960 . Beitrag zur Samenübertragung bei Ziegen, bei Anwendung des Tiefkühlferfahrens $\left(-79^{\circ} \mathrm{C}\right)$. Deutsche Tier. Wochenschr., 67, 127-129.

LyNGSet O., AAMdal J., Velle W., 1965 . Artificial insemination in the goat with deep frozen semen after hormonal synchronization of ostrus. Nord. Vel. Med., 17, 178-181.

Niwa T., ig6i. Communnication personnelle à Weissflog (voir thèse de Weissf log, ig6i).

Smiti A. U., P'OLGE C., I950. Storage of bull semen at low temperature. Vet. Rec., 62, 9-15.

Vlachos K., TskalofF P., I963. Die künstiche Besammung von Ziegen mit Tiefküklsperma. Berlin, Münch. Tierarz. Wochensch., 24, 49I-494.

WaIdE Y., NiWA T., I96r. Storage and insemination trials with frozen semen of goats. VIII Cong. internat. zootech., Hambourg.

Weissflog K., I961. Samenübertragung bei Ziegen, 12. Beitrag-Versuche Zur Samenkonservierung und Besamung unter Anwendung des Tiefkühlferfahrens. Disserl., Richard Gölze Instilut, Hannover.

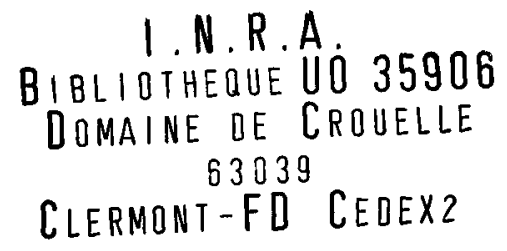

